\title{
The Linear 2- and 4-Arboricity of Complete Bipartite Graph $K_{m, n}$
}

\author{
Liancui Zuo, ${ }^{1}$ Bing Xue, ${ }^{2}$ and Shengjie $\mathrm{He}^{1}$ \\ ${ }^{1}$ College of Mathematical Science, Tianjin Normal University, Tianjin 300387, China \\ ${ }^{2}$ School of Mathematics, Shandong University, Jinan 250100, China \\ Correspondence should be addressed to Liancui Zuo; lczuo@163.com
}

Received 7 August 2013; Accepted 30 October 2013

Academic Editor: Jun-Ming Xu

Copyright (C) 2013 Liancui Zuo et al. This is an open access article distributed under the Creative Commons Attribution License, which permits unrestricted use, distribution, and reproduction in any medium, provided the original work is properly cited.

A linear $k$-forest of an undirected graph $G$ is a subgraph of $G$ whose components are paths with lengths at most $k$. The linear $k$-arboricity of $G$, denoted by $\operatorname{la}_{k}(G)$, is the minimum number of linear $k$-forests needed to decompose $G$. In case the lengths of paths are not restricted, we then have the linear arboricity of $G$, denoted by la $(G)$. In this paper, the exact value of the linear 2 - and 4 -arboricity of complete bipartite graph $K_{m, n}$ for some $m$ and $n$ is obtained.

\section{Introduction}

Throughout this paper, all graphs considered are finite, undirected, and simple. Let $\mathbb{N}$ represent the set of natural numbers and let $[a, b]$ denote the set $\{n \in \mathbb{N} \mid a \leq n \leq b\}$. A graph $G$ is $m$-partite ( $m \geq 2$ ), if it is possible to partition the vertex set $V(G)$ into $m$ independent sets $V_{1}, V_{2}, \ldots, V_{m}$ (called partite sets) such that every edge of $G$ joins the vertices in different partite sets. A complete $m$-partite graph is one that is simple and in which each vertex is joined to every vertex that is not in the same subset, which is denoted by $K_{n_{1}, n_{2}, \ldots, n_{m}}$ if $\left|V_{i}\right|=n_{i}$ for $i \in[1, m]$. For $m=2$, such graphs are called complete bipartite graphs and denoted by $K_{n_{1}, n_{2}}$. When $n_{1}=n_{2}=\cdots=n_{m}=n$, we denote $K_{n_{1}, n_{2}, \ldots, n_{m}}$ by $K_{m(n)}$, which is called balanced complete $m$-partite graph. For $m=2$, such graphs are called balanced complete bipartite graphs and are denoted by $K_{n, n}$. On the other hand, a graph with order $n$, in which any pair of different vertices are adjacent, is a complete graph, denoted by $K_{n}$. Other notations and terminology in the paper are the same as in [1].

A decomposition of a graph is a list of subgraphs such that each edge appears in exactly one subgraph in the list. If a graph $G$ has a decomposition $G_{1}, G_{2}, \ldots, G_{t}$, then we say that $G_{1}, G_{2}, \ldots, G_{t}$ decompose $G$ or $G$ can be decomposed into $G_{1}, G_{2}, \ldots, G_{t}$. Furthermore, a linear $k$-forest is a forest whose components are paths of length at most $k$. The linear $k$-arboricity of a graph $G$, denoted by $\operatorname{la}_{k}(G)$, is the least number of linear $k$-forests needed to decompose $G$.

The notion of linear $k$-arboricity of a graph was first introduced by Habib and Peroche [2]. It is a natural generalization of edge coloring. Clearly, a linear 1-forest is induced by a matching, and $\operatorname{la}_{1}(G)$ is the chromatic index $\chi^{\prime}(G)$ of a graph $G$. Moreover, the linear $k$-arboricity $\operatorname{la}_{k}(G)$ is also a refinement of the ordinary linear arboricity la $(G)$ (or $\mathrm{la}_{\infty}(G)$ ) which is the case when every component of each forest is a path with no length constraint. By the way, the notion of linear arboricity was introduced earlier by Harary in [3].

In 1982, Habib and Péroche [4] proposed the following conjecture for an upper bound on $\operatorname{la}_{k}(G)$.

Conjecture 1 (see [4]). If $G$ is a graph with maximum degree $\Delta(G)$ and $k \geq 2$, then

$$
\begin{aligned}
& l a_{k}(G) \\
& \quad \leq\left\{\begin{array}{l}
{\left[\frac{\Delta(G)|V(G)|}{2\lfloor k|V(G)| /(k+1)\rfloor}\right], \text { when } \Delta(G)=|V(G)|-1,} \\
{\left[\frac{\Delta(G)|V(G)|+1}{2\lfloor k|V(G)| /(k+1)\rfloor}\right],}
\end{array}\right.
\end{aligned}
$$


For $k=|V(G)|-1$, this is Akiyama's conjecture [5].

Conjecture 2 (see $[5])$. Consider $l a(G) \leq\lceil(\Delta(G)+1) / 2\rceil$.

So far, there have been a lot of results on the verification of Conjecture 1 in the literature, especially for graphs with particular structures, such as trees $[2,6,7]$, regular graphs $[8,9]$, planar graphs [10], and complete graphs [11-15]. The linear arboricity and the linear $k$-arboricity of cubic graphs are studied in recent years, and for more details, please read the papers [11, 16-20]. The linear 2-arboricity, the linear 3 -arboricity and the low bound of linear $k$-arboricity of balanced complete bipartite graph, and linear 3-arboricity of the balanced complete multipartite graph in $[13,15,21,22]$, respectively, are obtained. In 2010, Xue and Zuo obtained the linear $(n-1)$-arboricity of $K_{n(m)}$ in [23].

As for a low bound on $\mathrm{la}_{k}(G)$, since any vertex in a linear $k$-forest has degree at most 2 and a linear $k$-forest in a graph $G$ has at most $\lfloor k \cdot|V(G)| /(k+1)\rfloor$ edges, the following result is obvious.

Lemma 3 (see [15]). For any graph $G$ with maximum degree $\Delta(G)$

$$
l a_{k}(G) \geq \max \left\{\left\lceil\frac{\Delta(G)}{2}\right\rceil,\left\lceil\frac{|E(G)|}{\lfloor k \cdot|V(G)| /(k+1)\rfloor}\right\rceil\right\} .
$$

It is clear that the following lemma holds.

Lemma 4. If $G=G_{1} \cup G_{2} \cup \cdots \cup G_{n}$, then $l a_{k}(G) \leq l a_{k}\left(G_{1}\right)+$ $l a_{k}\left(G_{2}\right)+\cdots+l a_{k}\left(G_{n}\right)$.

In the following, we will study the linear 2- and 4-arboricity of the complete bipartite graph $K_{m, n}$.

\section{The Linear 2-Arboricity of Complete Bipartite Graph $K_{m, n}$}

Lemma 5. Consider $l a_{2}\left(K_{4,2}\right)=2$ and $l a_{2}\left(K_{4,3}\right)=l a_{2}\left(K_{5,2}\right)$ $=3$.

Proof. By Lemma 3, $\mathrm{la}_{2}\left(K_{4,2}\right) \geq 2, \mathrm{la}_{2}\left(K_{4,3}\right) \geq 3$, and $\mathrm{la}_{2}\left(K_{5,2}\right)$ $\geq 3$.

Let $K_{4,2}=\left(V_{1}, V_{2}\right)$, where $V_{1}=\left\{v_{i}: i \in[1,4]\right\}$ and $V_{2}=$ $\left\{u_{1}, u_{2}\right\}$. Clearly, $K_{4,2}$ can be decomposed into two linear 2forests: $\left\{v_{1} u_{1} v_{2}, v_{3} u_{2} v_{4}\right\}$ and $\left\{v_{1} u_{2} v_{2}, v_{3} u_{1} v_{4}\right\}$. Thus $\operatorname{la}_{2}\left(K_{4,2}\right)=$ 2 .

Let $K_{4,3}=\left(V_{1}^{\prime}, V_{2}^{\prime}\right)$, where $V_{1}^{\prime}=\left\{v_{i}: i \in[1,4]\right\}$ and $V_{2}^{\prime}=\left\{u_{j}: j \in[1,3]\right\}$. Clearly, $K_{4,3}$ can be decomposed into three linear 2-forests: $\left\{v_{1} u_{1} v_{2}, v_{3} u_{2} v_{4}\right\},\left\{v_{1} u_{2} v_{2}, v_{3} u_{3} v_{4}\right\}$, and $\left\{v_{1} u_{3} v_{2}, v_{3} u_{1} v_{4}\right\}$. Thus $\operatorname{la}_{2}\left(K_{4,3}\right)=3$.

Let $K_{5,2}=\left(V_{1}^{\prime \prime}, V_{2}^{\prime \prime}\right)$, where $V_{1}^{\prime \prime}=\left\{v_{i}: i \in[1,5]\right\}$ and $V_{2}^{\prime \prime}=$ $\left\{u_{1}, u_{2}\right\}$. Clearly, $K_{5,2}$ can be decomposed into three linear 2-forests: $\left\{v_{1} u_{1} v_{2}, v_{3} u_{2} v_{4}\right\},\left\{v_{1} u_{2} v_{2}, v_{4} u_{1} v_{5}\right\}$, and $\left\{v_{3} u_{1}, v_{5} u_{2}\right\}$. Thus $\operatorname{la}_{2}\left(K_{5,2}\right)=3$.

In the following, we mainly consider the complete bipartite graphs $K_{4 n, 2 n}, K_{4 n+1,2 n}$, and $K_{4 n, 2 n+1}$ in this section.

Let $G\left(V_{1}, V_{2}\right)$ be a bipartite graph with partite sets $V_{1}=$ $\left\{v_{10}, v_{11}, \ldots, v_{1(r-1)}\right\}$ and $V_{2}=\left\{v_{20}, v_{21}, \ldots, v_{2(s-1)}\right\}$. Suppose that $\left|V_{2}\right|=s \geq r=\left|V_{1}\right|$. In [15], Fu et al. defined the bipartite difference of an edge $v_{1 p} v_{2 q}$ in $G\left(V_{1}, V_{2}\right)$ as the value $(q-p)(\bmod s)$. It is not difficult to see that a set consisting of those edges in $G\left(V_{1}, V_{2}\right)$ with the same bipartite difference must be a matching. In particular, such a set is a perfect matching if $G\left(V_{1}, V_{2}\right)$ is a $K_{n, n}$. Furthermore, we can partition the edge set of $K_{n, n}$ into $n$ pairwise disjoint perfect matchings $M_{0}, M_{1}, \ldots, M_{n-1}$ such that $M_{i}$ is exactly the set of edges of bipartite difference $i$ in $K_{n, n}$ for $i \in[0, n-1]$.

Theorem 6. One has $l a_{2}\left(K_{4 n, 2 n}\right)=2 n$.

Proof. Let $G\left(V_{1}, V_{2}\right)=K_{4 n, 2 n}$ be a complete bipartite graph with partite sets $V_{1}$ and $V_{2}$, where $\left|V_{1}\right|=4 n$ and $\left|V_{2}\right|=2 n$. Next, we partition $V_{1}$ and $V_{2}$ into $n$ vertex sets $V_{1 i}$ and $V_{2 i}$, respectively, such that $\left|V_{1 i}\right|=4$ and $\left|V_{2 i}\right|=2$ for $i \in[0, n-1]$.

For each $V_{1 i}(i \in[0, n-1])$, we identify 4 vertices in $V_{1 i}$ and denote a new vertex by $v_{1[i]}$. For each $V_{2 i}$, we identify 2 vertices in $V_{2 i}$ and denote a new vertex by $v_{2[i]}$. Then we obtain a balanced complete bipartite graph $H=K_{n, n}$ with vertex sets $V_{1}^{\prime}=\left\{v_{1[i]}: i \in[0, n-1]\right\}$ and $V_{2}^{\prime}=\left\{v_{2[i]}: i \in[0, n-\right.$ $1]$ \}. The edge set of $K_{n, n}$ can be partitioned into $n$ pairwise disjoint perfect matchings $M_{0}, M_{1}, \ldots, M_{n-1}$ such that $M_{j}$ is exactly the set of edges of bipartite difference $j$ in $K_{n, n}$ for $j \in[0, n-1]$. Every edge in $M_{j}$ corresponds to a subgraph of $K_{4 n, 2 n}$ isomorphic to $K_{4,2}$. Since $\mathrm{la}_{2}\left(K_{4,2}\right)=2$ by Lemma 5 , each $M_{j}$ corresponds to two linear 2 -forests in $K_{4 n, 2 n}$. Thus $\operatorname{la}_{2}\left(K_{4 n, 2 n}\right) \leq 2 n$ by Lemma 4 . Applying Lemma 3, we have $\operatorname{la}_{2}\left(K_{4 n, 2 n}\right) \geq 2 n$. Hence la $2\left(K_{4 n, 2 n}\right)=2 n$.

Corollary 7. One has $l a_{2}\left(K_{4 n, 2 n-1}\right)=l a_{2}\left(K_{4 n-1,2 n}\right)=2 n$.

Proof. The results can be obtained by Lemma 3 and Theorem 6 immediately.

Theorem 8. $l a_{2}\left(K_{4 n, 2 n+1}\right)=2 n+1$, where $n$ is odd.

Proof. Let $G\left(V_{1}, V_{2}\right)=K_{2 n+1,4 n}$ be a complete bipartite graph with partite sets $V_{1}$ and $V_{2}$, where $\left|V_{1}\right|=2 n+1$ and $\left|V_{2}\right|=4 n$. Next, we partition $V_{1}$ into $n+1$ vertex sets $V_{1 j}(j \in[0, n])$ and partition $V_{2}$ into $n$ vertex sets $V_{2 i}(i \in[0, n-1])$, respectively, such that $\left|V_{1 i}\right|=2,\left|V_{2 i}\right|=4$ for $i \in[0, n-1]$, and $\left|V_{1 n}\right|=$ $|\{u\}|=1$.

For each $V_{1 i}(i \in[0, n-1])$, we identify 2 vertices in $V_{1 i}$ and denote a new vertex by $v_{1[i]}$. For each $V_{2 i}(i \in[0, n-$ $1]$ ), we identify 4 vertices in $V_{2 i}$ and denote a new vertex by $v_{2[i]}$. Then we obtain a complete bipartite graph $H=K_{n+1, n}$ with partite sets $V_{1}^{\prime}=\left\{v_{1[i]}: i \in[0, n-1]\right\} \cup\{u\}$ and $V_{2}^{\prime}=$ $\left\{v_{2[i]}: i \in[0, n-1]\right\}$. Let $H_{1}\left(V, V_{2}^{\prime}\right)=K_{n, n}$ be a balanced complete bipartite graph with partite sets $V=V_{1}^{\prime} \backslash\{u\}=$ $\left\{v_{1[i]}: i \in[0, n-1]\right\}$ and $V_{2}^{\prime}=\left\{v_{2[j]}: j \in[0, n-1]\right\}$. Let $H_{2}=K_{n, 1}$ be a complete bipartite graph with vertex sets $V_{2}^{\prime}=$ $\left\{v_{2[i]}: i \in[0, n-1]\right\}$ and $\{u\}$. In fact, $H_{2}=K_{n, 1}$ is a star $S_{n+1}$ with the center $u$. Thus, $H=H_{1} \cup H_{2}$. Since the edge set of $H_{1}\left(V, V_{2}^{\prime}\right)=K_{n, n}$ can be partitioned into $n$ pairwise disjoint perfect matchings $M_{0}, M_{1}, \ldots, M_{n-1}$ such that $M_{j}$ is exactly the set of edges of bipartite difference $j$ in $K_{n, n}$ for $j \in[0, n-1]$ and $n$ is odd, the edge set of $H=K_{n+1, n}$ can be partitioned 
into $n$ pairwise disjoint edge sets $M_{0}^{\prime}, M_{1}^{\prime}, \ldots, M_{n-1}^{\prime}$ such that $M_{j}^{\prime}=M_{j} \cup\left\{u v_{2[2 j]}\right\}(j \in[0, n-1])$ which consists of a 2-path $u v_{2[2 j]} v_{1[j]}$ and $n-1$ isolated edges $v_{1[i]} v_{2[i+j]}(i \in[0, j-1] \cup$ $[j+1, n-1])$, where $2 j, i+j$ are taken modulo $n$.

Thus, each edge $v_{1[i]} v_{2[i+j]}(i \in[0, j-1] \cup[j+1, n-1])$ in $M_{j}^{\prime}$ corresponds to a subgraph isomorphic to $K_{4,2}$ and the 2-path $u v_{2[2 j]} v_{1[j]}$ corresponds to a subgraph isomorphic to $K_{4,3}$ with partition $\left(V_{2(2 j)}, V_{1 j} \cup\{u\}\right)$ in $K_{2 n+1,4 n}$. Let

$$
\begin{aligned}
V_{1 j} & =\left\{v_{1[j] 0}, v_{1[j] 1}\right\}, \\
V_{2(2 j)} & =\left\{v_{2[2 j] 0}, v_{2[2 j] 1}, v_{2[2 j] 2}, v_{2[2 j] 3}\right\},
\end{aligned}
$$

where the indices $2 j$ are taken modulo $n$.

After taking away the four edges $v_{2[2 j] 0} v_{1[j] 0}, v_{2[2 j] 1} v_{1[j] 0}$, $v_{2[2 j] 2} v_{1[j] 1}$, and $v_{2] 2 j] 3} v_{1[j] 1}$ from each $K_{4,3}$ to which the $2-$ path $u v_{2[2 j]} v_{1[j]}$ corresponds, each $M_{j}^{\prime}$ corresponds to two linear 2 -forests of $K_{4 n, 2 n+1}$ by Lemma 5 . The edges that we take away form one linear 2 -forest. Hence $\mathrm{la}_{2}\left(K_{2 n+1,4 n}\right) \leq 2 n+1$ by Lemma 4. Since $\mathrm{la}_{2}\left(K_{2 n+1,4 n}\right) \geq 2 n+1$ by Lemma 3, we have $\mathrm{la}_{2}\left(K_{2 n+1,4 n}\right)=2 n+1$.

Corollary 9. $l a_{2}\left(K_{4 n-1,2 n+1}\right)=2 n+1$, where $n$ is odd.

Proof. The results can be obtained by Lemma 3 and Theorem 8 immediately.

Theorem 10. $l a_{2}\left(K_{4 n+1,2 n}\right)=2 n+1$, where $n$ is odd.

Proof. The proof is similar to Theorem 8. Let $G\left(V_{1}, V_{2}\right)=$ $K_{4 n+1,2 n}$ be a complete bipartite graph with partite sets $V_{1}$ and $V_{2}$, where $\left|V_{1}\right|=4 n+1$ and $\left|V_{2}\right|=2 n$. Next, we partition $V_{2}$ into $n$ vertex sets $V_{2 i}(i \in[0, n-1])$ and partition $V_{1}$ into $n+1$ vertex sets $V_{1 i}(i \in[0, n])$, respectively, such that $\left|V_{2 i}\right|=2$, $\left|V_{1 i}\right|=4$ for $i \in[0, n-1]$, and $\left|V_{1 n}\right|=|\{u\}|=1$.

For each $V_{1 i}(i \in[0, n-1])$, we identify 4 vertices in $V_{1 i}$ and denote a new vertex by $v_{1[i]}$. For each $V_{2 i}(i \in[0, n-1])$, we identify 2 vertices in $V_{2 i}$ and denote a new vertex by $v_{2[i]}$. Then we obtain a complete bipartite graph $H=K_{n+1, n}$ with partite sets $V_{1}^{\prime}=\left\{v_{1[i]}: i \in[0, n-1]\right\} \cup\{u\}$ and $V_{2}^{\prime}=\left\{v_{2[i]}: i \in\right.$ $[0, n-1]\}$. Let $H_{1}\left(V, V_{2}^{\prime}\right)=K_{n, n}$ be a balanced complete bipartite graph with vertex sets $V=V_{1}^{\prime} \backslash\{u\}=\left\{v_{1[i]}: i \in[0, n-1]\right\}$ and $V_{2}^{\prime}=\left\{v_{2[i]}: i \in[0, n-1]\right\}$. Let $H_{2}=K_{n, 1}$ be a complete bipartite graph with partite sets $V_{2}^{\prime}=\left\{v_{2[i]}: i \in[0, n-1]\right\}$ and $\{u\}$. In fact, $H_{2}=K_{n, 1}$ is a star $S_{n+1}$ with the center $u$. Thus, $H=H_{1} \cup H_{2}$. Since the edge set of $H_{1}\left(V, V_{2}^{\prime}\right)=K_{n, n}$ can be partitioned into $n$ pairwise disjoint perfect matchings $M_{0}, M_{1}, \ldots, M_{n-1}$ such that $M_{j}$ is exactly the set of edges of bipartite difference $j$ in $K_{n, n}$ for $j \in[0, n-1]$ and $n$ is odd, the edge set of $H=K_{n+1, n}$ can be partitioned into $n$ pairwise disjoint edge sets $M_{0}^{\prime}, M_{1}^{\prime}, \ldots, M_{n-1}^{\prime}$ such that $M_{j}^{\prime}=M_{j} \cup$ $\left\{u v_{2[2 j]}\right\}(j \in[0, n-1])$ which consists of a 2-path $u v_{2[2 j]} v_{1[j]}$ and $n-1$ isolated edges $v_{1[i]} v_{2[i+j]}(i \in[0, j-1] \cup[j+1, n-1])$, where $2 j$ and $i+j$ are taken modulo $n$.

Thus, each edge $v_{1[i]} v_{2[i+j]}(i \in[0, j-1] \cup[j+1, n-1])$ in $M_{j}^{\prime}$ corresponds to a subgraph isomorphic to $K_{4,2}$ and the 2-path $u v_{2[2 j]} v_{1[j]}$ corresponds to a subgraph isomorphic to $K_{5,2}$ with partition $\left(V_{1 j} \cup\{u\}, V_{2(2 j)}\right)$ in $K_{4 n+1,2 n}$. Let

$$
\begin{aligned}
V_{2(2 j)} & =\left\{v_{2[2 j] 0}, v_{2[2 j] 1}\right\}, \\
V_{1 j} & =\left\{v_{1[j] 0}, v_{1[j] 1}, v_{1[j] 2}, v_{1[j] 3}\right\},
\end{aligned}
$$

where the indices $2 j$ are taken modulo $n$.

After taking away the two edges $v_{1[j] 0} v_{2[2 j] 1}, v_{1[j] 1} v_{2[2 j] 3}$ from each $K_{5,2}$ to which the 2-path $u v_{2[2 j]} v_{1[j]}$ corresponds, each $M_{j}^{\prime}$ corresponds to two linear 2-forests of $K_{4 n+1,2 n}$ by Lemma 5. The edges that we take away form one linear 2forest. Hence $\mathrm{la}_{2}\left(K_{4 n+1,2 n}\right) \leq 2 n+1$ by Lemma 4 . Since $\mathrm{la}_{2}\left(K_{4 n+1,2 n}\right) \geq 2 n+1$ by Lemma 3 , we obtain $\mathrm{la}_{2}\left(K_{4 n+1,2 n}\right)=$ $2 n+1$.

\section{The Linear 4-Arboricity of Complete Bipartite Graph $K_{m, n}$}

Lemma 11. One has $l a_{4}\left(K_{6,4}\right)=3$.

Proof. By Lemma 3, $\mathrm{la}_{4}\left(K_{6,4}\right) \geq 3$. In the following, $K_{6,4}$ can be partitioned into three linear 4-forests: let $K_{6,4}=\left(V_{1}, V_{2}\right)$, where $V_{1}=\left\{v_{i}: i \in[1,6]\right\}$ and $V_{2}=\left\{u_{j}: j \in[1,4]\right\}$. Clearly, $K_{6,4}$ can be decomposed into three linear 4forests $\left\{v_{1} u_{1} v_{2} u_{2} v_{3}, v_{4} u_{3} v_{5} u_{4} v_{6}\right\},\left\{v_{2} u_{3} v_{1} u_{4} v_{3}, v_{4} u_{1} v_{6} u_{2} v_{5}\right\}$, and $\left\{v_{1} u_{2} v_{4} u_{4} v_{2}, v_{5} u_{1} v_{3} u_{3} v_{6}\right\}$ (see Figure 1).

Thus $\operatorname{la}_{4}\left(K_{6,4}\right)=3$.

Lemma 12. $l a_{4}\left(K_{6,5}\right)=4$.

Proof. By Lemma 3, $\mathrm{la}_{4}\left(K_{6,5}\right) \geq 4$. In the following, we partition the edge set of $K_{6,5}$ into four linear 4-forests: let $K_{6,5}=\left(V_{1}, V_{2}\right)$, where $V_{1}=\left\{v_{i}: i \in[1,6]\right\}$ and $V_{2}=\left\{u_{j}: j\right.$ $\in[1,5]\}$. Clearly, $K_{6,5}$ can be decomposed into four linear 4-forests $\left\{v_{1} u_{1} v_{2} u_{2}, v_{3} u_{3} v_{4} u_{4}, v_{5} u_{5} v_{6}\right\},\left\{v_{1} u_{5} v_{2}, u_{1} v_{3} u_{2} v_{4}\right.$, $\left.u_{3} v_{5} u_{4} v_{6}\right\},\left\{v_{1} u_{3} v_{2} u_{4}, v_{3} u_{5} v_{4}, u_{1} v_{5} u_{2} v_{6}\right\}$, and $\left\{u_{2} v_{1} u_{4} v_{3}, v_{4} u_{1}\right.$ $\left.v_{6} u_{3}\right\}$ (see Figure 2).

Thus $\operatorname{la}_{4}\left(K_{6,5}\right)=4$.

Lemma 13. $l a_{4}\left(K_{7,4}\right)=4$.

Proof. By Lemma 3, $\mathrm{la}_{4}\left(K_{7,4}\right) \geq 4$. In the following, we partition the edge set of $K_{7,4}$ into four linear 4-forests: let $K_{7,4}=\left(V_{1}, V_{2}\right)$, where $V_{1}=\left\{v_{i}: i \in[1,7]\right\}$ and $V_{2}=\left\{u_{j}\right.$ : $j \in[1,4]\} . K_{7,4}$ can be decomposed into four linear 4 -forests $\left\{v_{1} u_{1} v_{2}, v_{3} u_{2} v_{4}, v_{5} u_{3} v_{6} u_{4} v_{7}\right\}, \quad\left\{v_{1} u_{4} v_{2}, v_{3} u_{1} v_{4} u_{3} v_{7}, v_{5} u_{2} v_{6}\right\}$, $\left\{v_{1} u_{3} v_{2}, v_{3} u_{4} v_{4}, v_{6} u_{1} v_{7} u_{2}\right\}$, and $\left\{v_{1} u_{2} v_{2}, u_{1} v_{5} u_{4}, v_{3} u_{3}\right\}$ (see Figure 3).

Thus $\mathrm{la}_{4}\left(K_{7,4}\right)=4$.

In the following, we mainly consider the complete bipartite graphs $K_{6 n, 4 n}, K_{6 n+1,4 n}$, and $K_{6 n, 4 n+1}$.

Theorem 14. One has $l a_{4}\left(K_{6 n, 4 n}\right)=3 n$.

Proof. Let $G\left(V_{1}, V_{2}\right)=K_{6 n, 4 n}$ be a complete bipartite graph with partite sets $V_{1}$ and $V_{2}$, where $\left|V_{1}\right|=6 n$ and $\left|V_{2}\right|=4 n$. 


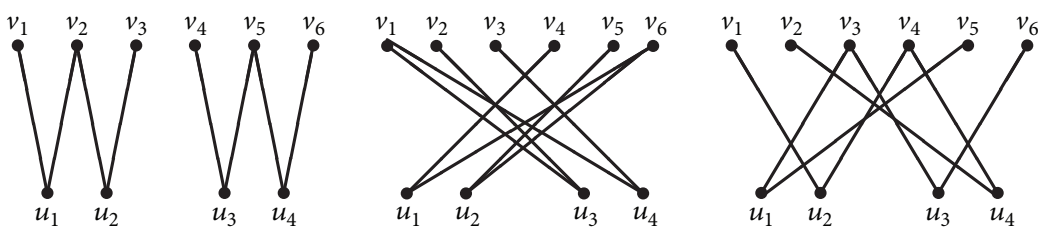

FIgURE 1: The decomposition of linear 4 -forests of $k_{6,4}$.
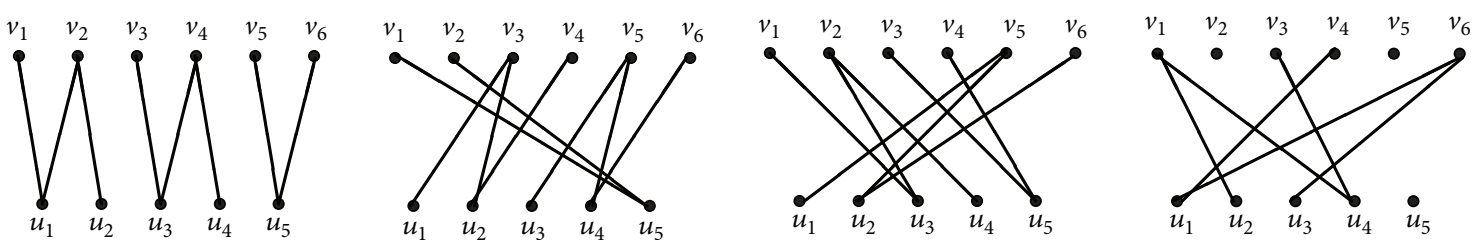

FIgURE 2: The decomposition of linear 4 -forests of $K_{6,5}$.

Next, we partition $V_{1}$ and $V_{2}$ into $n$ vertex sets $V_{1 i}$ and $V_{2 i}$, respectively, such that $\left|V_{1 i}\right|=6$ and $\left|V_{2 i}\right|=4$ for $i \in[0, n-1]$.

For each $V_{1 i}(i \in[0, n-1])$, we identify 6 vertices in $V_{1 i}$ and denote a new vertex by $v_{1[i]}$. For each $V_{2 i}$, we identify 4 vertices in $V_{2 i}$ and denote a new vertex by $v_{2[i]}$. Then we obtain a balanced complete bipartite graph $H=K_{n, n}$ with vertex sets $V_{1}^{\prime}=\left\{v_{1[i]}: i \in[0, n-1]\right\}$ and $V_{2}^{\prime}=\left\{v_{2[i]}: i \in[0, n-\right.$ $1]\}$. The edge set of $K_{n, n}$ can be partitioned into $n$ pairwise disjoint perfect matchings $M_{0}, M_{1}, \ldots, M_{n-1}$ such that $M_{j}$ is exactly the set of edges of bipartite difference $j$ in $K_{n, n}$ for $j \in[0, n-1]$. Every edge in $M_{j}$ corresponds to a subgraph of $K_{6 n, 4 n}$ isomorphic to $K_{6,4}$. Since $\mathrm{la}_{4}\left(K_{6,4}\right)=3$ by Lemma 11, each $M_{j}$ corresponds to three linear 4 -forests in $K_{6 n, 4 n}$. Thus $\mathrm{la}_{4}\left(K_{6 n, 4 n}\right) \leq 3 n$ by Lemma 11. Applying Lemma 3, we have

$$
\operatorname{la}_{4}\left(K_{6 n, 4 n}\right) \geq\left\lceil\frac{6 n \cdot 4 n}{\lfloor 4 \cdot(6 n+4 n) / 5\rfloor}\right\rceil=3 n .
$$

Hence $\operatorname{la}_{4}\left(K_{6 n, 4 n}\right)=3 n$.

Corollary 15. Consider $l a_{4}\left(K_{6 n, 4 n-1}\right)=l a_{4}\left(K_{6 n-1,4 n}\right)=3 n$.

Proof. The results can be obtained by Lemma 3 and Theorem 14 immediately.

Theorem 16. $l a_{4}\left(K_{4 n+1,6 n}\right)=3 n+1$, where $n$ is odd.

Proof. Let $G\left(V_{1}, V_{2}\right)=K_{4 n+1,6 n}$ be a complete bipartite graph with partite sets $V_{1}$ and $V_{2}$, where $\left|V_{1}\right|=4 n+1$ and $\left|V_{2}\right|=6 n$. Next, we partition $V_{1}$ into $n+1$ vertex sets $V_{1 i}(i \in[0, n])$ and partition $V_{2}$ into $n$ vertex sets $V_{2 j}$, respectively, such that $\left|V_{1 j}\right|=4,\left|V_{2 j}\right|=6$ for $j \in[0, n-1]$, and $\left|V_{1 n}\right|=|\{u\}|=1$.

For each $V_{1 i}(i \in[0, n-1])$, we identify 4 vertices in $V_{1 i}$ and denote a new vertex by $v_{1[i]}$. For each $V_{2 i}(i \in[0, n-1])$, we identify 6 vertices in $V_{2 i}$ and denote a new vertex by $v_{2[i]}$. Then we obtain a complete bipartite graph $H=K_{n+1, n}$ with partite sets $V_{1}^{\prime}=\left\{v_{1[i]}: i \in[0, n-1]\right\} \cup\{u\}$ and $V_{2}^{\prime}=\left\{v_{2[i]}\right.$ : $i \in[0, n-1]\}$. Let $H_{1}\left(V, V_{2}^{\prime}\right)=K_{n, n}$ be a balanced complete bipartite graph with partite sets $V=V_{1}^{\prime} \backslash\{u\}=\left\{v_{1[i]}: i \in\right.$ $[0, n-1]\}$ and $V_{2}^{\prime}$. Let $H_{2}=K_{n, 1}$ be a complete bipartite graph with vertex sets $V_{2}^{\prime}$ and $\{u\}$. In fact, $H_{2}=K_{n, 1}$ is a star $S_{n+1}$ with the center $u$. Thus, $H=H_{1} \cup H_{2}$. Since the edge set of $H_{1}\left(V, V_{2}^{\prime}\right)=K_{n, n}$ can be partitioned into $n$ pairwise disjoint perfect matchings $M_{0}, M_{1}, \ldots, M_{n-1}$ such that $M_{j}$ is exactly the set of edges of bipartite difference $j$ in $K_{n, n}$ for $j \in[0, n-1]$ and $n$ is odd, the edge set of $H=K_{n+1, n}$ can be partitioned into $n$ pairwise disjoint edge sets $M_{0}^{\prime}, M_{1}^{\prime}, \ldots, M_{n-1}^{\prime}$ such that $M_{j}^{\prime}=M_{j} \cup\left\{u v_{2[2 j]}\right\} \quad(j \in[0, n-1])$ which consists of a 2-path $u v_{2[2 j]} v_{1[j]}$ and $n-1$ isolated edges $v_{1[i]} v_{2[i+j]}(i \in[0, j-1] \cup$ $[j+1, n-1])$, where $2 j, i+j$ are taken modulo $n$.

Thus, each edge $v_{1[i]} v_{2[i+j]}(i \in[0, j-1] \cup[j+1, n-1])$ in $M_{j}^{\prime}$ corresponds to a subgraph isomorphic to $K_{6,4}$ and the 2-path $u v_{2[2 j]} v_{1[j]}$ corresponds to a subgraph isomorphic to $K_{6,5}$ with partition $\left(V_{2(2 j)}, V_{1 j} \cup\{u\}\right)$ in $K_{6 n, 4 n+1}$, where $2 j$ is taken modulo $n$. Let

$$
\begin{aligned}
V_{1 j} & =\left\{v_{1[j] 0}, v_{1[j] 1}, v_{1[j] 2}, v_{1[j] 3}\right\}, \\
V_{2(2 j)} & =\left\{v_{2[2 j] 0}, v_{2[2 j] 1}, \ldots, v_{2[2 j] 5}\right\},
\end{aligned}
$$

where the indices $2 j$ are taken modulo $n$.

After taking away the six edges $v_{2[2 j] 0} v_{1[j] 1}, v_{2[2 j] 0} v_{1[j] 3}$, $v_{2[2 j] 2} v_{1[j] 3}, v_{2[2 j] 3} v_{1[j] 0}, v_{2[2 j] 5} v_{1[j] 0}$, and $v_{2[2 j] 5} v_{1[j] 2}$ from each $K_{6,5}$ to which the 2-path $u v_{2[2 j]} v_{1[j]}$ corresponds, each $M_{j}^{\prime}$ corresponds to three linear 4 -forests of $K_{6 n, 4 n+1}$ by Lemma 12. The edges that we take away form one linear 4 -forest (see Figure 2). Hence $\operatorname{la}_{4}\left(K_{6 n, 4 n+1}\right) \leq 3 n+1$ by Lemma 11. Since

$$
\begin{aligned}
\operatorname{la}_{4}\left(K_{6 n, 4 n+1}\right) & \geq\left\lceil\frac{6 n(4 n+1)}{\lfloor 4 \cdot(6 n+4 n+1) / 5\rfloor}\right\rceil \\
& =3 n+1
\end{aligned}
$$

by Lemma 3 , we have $\operatorname{la}_{4}\left(K_{6 n, 4 n+1}\right)=3 n+1$.

Corollary 17. $l a_{4}\left(K_{6 n-1,4 n+1}\right)=3 n+1$, where $n$ is odd.

Proof. The results can be obtained by Lemma 3 and Theorem 16 immediately. 

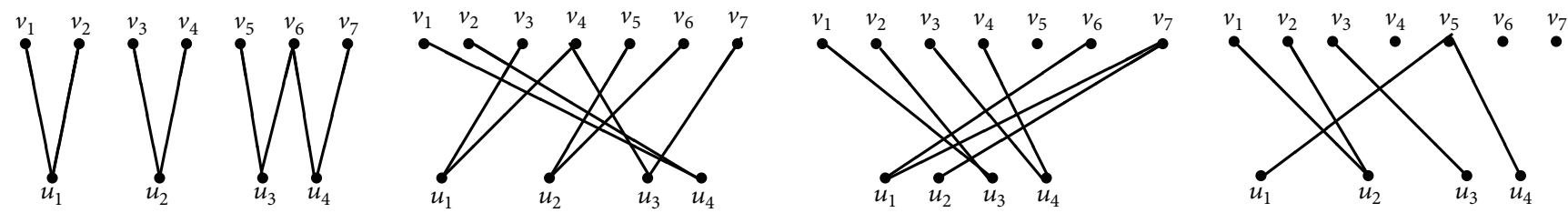

FIgURE 3: The decomposition of linear 4 -forests of $K_{7,4}$.

Theorem 18. $l a_{4}\left(K_{6 n+1,4 n}\right)=3 n+1$, where $n$ is odd

Proof. The proof is similar to Theorem 16. Let $G\left(V_{1}, V_{2}\right)=$ $K_{6 n+1,4 n}$ be a complete bipartite graph with partite sets $V_{1}$ and $V_{2}$, where $\left|V_{1}\right|=6 n+1$ and $\left|V_{2}\right|=4 n$. Next, we partition $V_{2}$ into $n$ vertex subsets $V_{2 i}(i \in[0, n-1])$ and partition $V_{1}$ into $n+1$ vertex subsets $V_{1 i}(i \in[0, n])$, respectively, such that $\left|V_{2 i}\right|=4,\left|V_{1 i}\right|=6$ for $i \in[0, n-1]$, and $\left|V_{1 n}\right|=|\{u\}|=1$.

For each $V_{1 i}(i \in[0, n-1])$, we identify 6 vertices in $V_{1 i}$ and denote a new vertex by $v_{1[i]}$. For each $V_{2 i}(i \in[0, n-1])$, we identify 4 vertices in $V_{2 i}$ and denote a new vertex by $v_{2[i]}$. Then we obtain a complete bipartite graph $H=K_{n+1, n}$ with partite sets $V_{1}^{\prime}=\left\{v_{1[i]}: i \in[0, n-1]\right\} \cup\{u\}$ and $V_{2}^{\prime}=\left\{v_{2[i]}\right.$ : $i \in[0, n-1]\}$. Let $H_{1}\left(V, V_{2}^{\prime}\right)=K_{n, n}$ be a balanced complete bipartite graph with vertex sets $V=V_{1}^{\prime} \backslash\{u\}=\left\{v_{1[i]}: i \epsilon\right.$ $[0, n-1]\}$ and $V_{2}^{\prime}=\left\{v_{2[i]}: i \in[0, n-1]\right\}$. Let $H_{2}=K_{n, 1}$ be a complete bipartite graph with partite sets $V_{2}^{\prime}=\left\{v_{2[i]}\right.$ : $i \in[0, n-1]\}$ and $\{u\}$. In fact, $H_{2}=K_{n, 1}$ is a star $S_{n+1}$ with the center $u$. Thus, $H=H_{1} \cup H_{2}$. Since the edge set of $H_{1}\left(V, V_{2}^{\prime}\right)=K_{n, n}$ can be partitioned into $n$ pairwise disjoint perfect matchings $M_{0}, M_{1}, \ldots, M_{n-1}$ such that $M_{j}$ is exactly the set of edges of bipartite difference $j$ in $K_{n, n}$ for $j \in[0, n-1]$ and $n$ is odd, the edge set of $H=K_{n+1, n}$ can be partitioned into $n$ pairwise disjoint edge sets $M_{0}^{\prime}, M_{1}^{\prime}, \ldots, M_{n-1}^{\prime}$ such that $M_{j}^{\prime}=M_{j} \cup\left\{u v_{2[2 j]}\right\}(j \in[0, n-1])$ which consists of a 2-path $u v_{2[2 j]} v_{1[j]}$ and $n-1$ isolated edges $v_{1[i]} v_{2[i+j]}(i \in$ $[0, j-1] \cup[j+1, n-1])$, where $2 j, i+j$ are taken modulo $n$.

Thus, each edge $v_{1[i]} v_{2[i+j]}(i \in[0, j-1] \cup[j+1, n-1])$ in $M_{j}^{\prime}$ corresponds to a subgraph isomorphic to $K_{6,4}$ and the 2-path $u v_{2[2 j]} v_{1[j]}$ corresponds to a subgraph isomorphic to $K_{7,4}$ with partition $\left(V_{1 j} \cup\{u\}, V_{2(2 j)}\right)$ in $K_{6 n+1,4 n}$, where $2 j$ is taken modulo $n$. Let

$$
\begin{aligned}
V_{2(2 j)} & =\left\{v_{2[2 j] 0}, v_{2[2 j] 1}, v_{2[2 j] 2}, v_{2[2 j] 3}\right\}, \\
V_{1 j} & =\left\{v_{1[j] 0}, v_{1[j] 1}, \ldots, v_{1[j] 5}\right\},
\end{aligned}
$$

where the indices $2 j$ are taken modulo $n$.

After taking away five edges $v_{1[j] 0} v_{2[2 j] 1}, v_{1[j] 1} v_{2[2 j] 1}, v_{1[j] 2}$ $v_{2[2 j] 2}, v_{1[j] 4} v_{2[2 j] 0}$, and $v_{1[j] 4} v_{2[2 j] 3}$ from each $K_{7,4}$ to which the 2-path $u v_{2[2 j]} v_{1[j]}$ corresponds, where the indices $2 j$ are taken modulo $n$, each $M_{j}^{\prime}$ corresponds to three linear 4 -forests of $K_{6 n+1,4 n}$ by Lemma 13. The edges that we take away form one linear 4-forest (see Figure 3). Hence $\mathrm{la}_{4}\left(K_{6 n+1,4 n}\right) \leq 3 n+1$ by Lemma 11. Since

$$
\operatorname{la}_{4}\left(K_{6 n+1,4 n}\right) \geq\left\lceil\frac{(6 n+1) 4 n}{\lfloor 4 \cdot(6 n+1+4 n) / 5\rfloor}\right\rceil=3 n+1
$$

by Lemma 3, we obtain $\mathrm{la}_{4}\left(K_{6 n+1,4 n}\right)=3 n+1$.

Corollary 19. $l a_{4}\left(K_{6 n+1,4 n-1}\right)=3 n+1$, where $n$ is odd.

Proof. The results can be obtained by Lemma 3 and Theorem 18 immediately.

\section{Acknowledgment}

This work is supported by NSFC for youth with code of 61103073.

\section{References}

[1] D. B. West, Introduction to Graph Theory, Prentice Hall, Upper Saddle River, NJ, USA, 2nd edition, 2001.

[2] M. Habib and B. Peroche, "La $k$-arboricité linéaire des arbres," Annales Polonici Mathematici, vol. 17, pp. 307-317, 1983.

[3] F. Harary, "Covering and packing in graphs. I," Annals of the New York Academy of Sciences, vol. 175, pp. 198-205, 1970.

[4] M. Habib and B. Péroche, "Some problems about linear arboricity," Discrete Mathematics, vol. 41, no. 2, pp. 219-220, 1982.

[5] J. Akiyama, Three developing topics in graph theory [doctoral dissertation], University of Toyo, 1980.

[6] G. J. Chang, "Algorithmic aspects of linear $k$-arboricity," Taiwanese Journal of Mathematics, vol. 3, no. 1, pp. 73-81, 1999.

[7] G. J. Chang, B.-L. Chen, H.-L. Fu, and K.-C. Huang, "Linear karboricities on trees," Discrete Applied Mathematics, vol. 103, no. 1-3, pp. 281-287, 2000.

[8] R. E. L. Aldred and N. C. Wormald, "More on the linear $k$-arboricity of regular graphs," The Australasian Journal of Combinatorics, vol. 18, pp. 97-104, 1998.

[9] N. Alon, V. J. Teague, and N. C. Wormald, "Linear arboricity and linear $k$-arboricity of regular graphs," Graphs and Combinatorics, vol. 17, no. 1, pp. 11-16, 2001.

[10] K.-W. Lih, L.-D. Tong, and W.-F. Wang, "The linear 2-arboricity of planar graphs," Graphs and Combinatorics, vol. 19, no. 2, pp. 241-248, 2003.

[11] J.-C. Bermond, J.-L. Fouquet, M. Habib, and B. Péroche, "On linear k-arboricity," Discrete Mathematics, vol. 52, no. 2-3, pp. 123-132, 1984.

[12] B.-L. Chen, H.-L. Fu, and K. C. Huang, "Decomposing graphs into forests of paths with size less than three," The Australasian Journal of Combinatorics, vol. 3, pp. 55-73, 1991. 
[13] B.-L. Chen and K.-C. Huang, "On the linear $k$-arboricity of $K_{n}$ and $K_{n, n}$ " Discrete Mathematics, vol. 254 , no. $1-3$, pp. 51-61, 2002.

[14] C.-H. Yen and H.-L. Fu, "Linear 2-arboricity of the complete graph," Taiwanese Journal of Mathematics, vol. 14, no. 1, pp. 273286, 2010.

[15] H.-L. Fu, K.-C. Huang, and C.-H. Yen, “The linear 3-arboricity of $K_{n, n}$ and $K_{n}$," Discrete Mathematics, vol. 308, no. 17, pp. 38163823, 2008.

[16] R. Berke and T. Szabó, "Relaxed two-coloring of cubic graphs," Journal of Combinatorial Theory B, vol. 97, no. 4, pp. 652-668, 2007.

[17] J.-L. Fouquet, H. Thuillier, J.-M. Vanherpe, and A. P. Wojda, "On isomorphic linear partitions in cubic graphs," Discrete Mathematics, vol. 309, no. 22, pp. 6425-6433, 2009.

[18] B. Jackson and N. C. Wormald, "On the linear $k$-arboricity of cubic graphs," Discrete Mathematics, vol. 162, no. 1-3, pp. 293297, 1996.

[19] C. Thomassen, "Two-coloring the edges of a cubic graph such that each monochromatic component is a path of length at most 5," Journal of Combinatorial Theory B, vol. 75, no. 1, pp. 100-109, 1999.

[20] A. Vesel and J. Žerovnik, "On the linear $k$-arboricity of cubic graphs," International Journal of Computer Mathematics, vol. 75, no. 4, pp. 431-444, 2000.

[21] H.-L. Fu and K. C. Huang, "The linear $k$-arboricity of complete bipartite graphs," Ars Combinatoria, vol. 38, pp. 309-318, 1994.

[22] C.-H. Yen and H.-L. Fu, "Linear 3-arboricity of the balanced complete multipartite graph," Journal of Combinatorial Mathematics and Combinatorial Computing, vol. 60, pp. 33-46, 2007.

[23] B. Xue and L. Zuo, "On the linear $(n-1)$-arboricity of $K_{n(m)}$," Discrete Applied Mathematics, vol. 158, no. 14, pp. 1546-1550, 2010. 


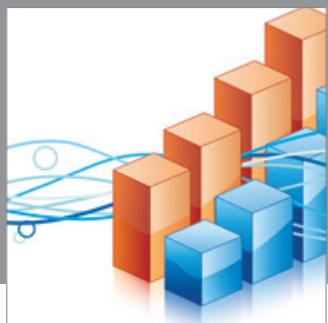

Advances in

Operations Research

mansans

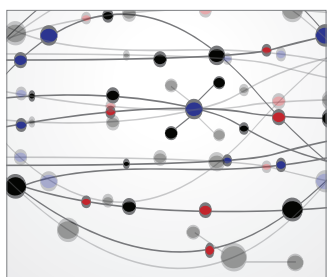

The Scientific World Journal
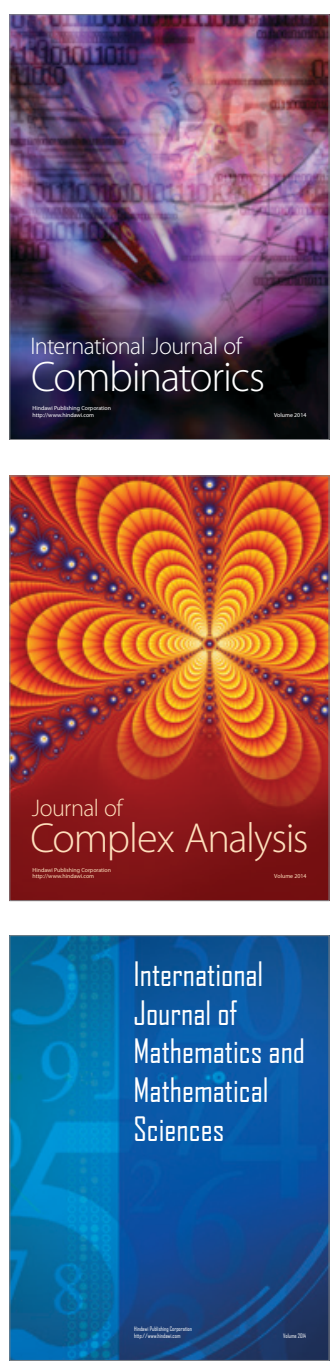
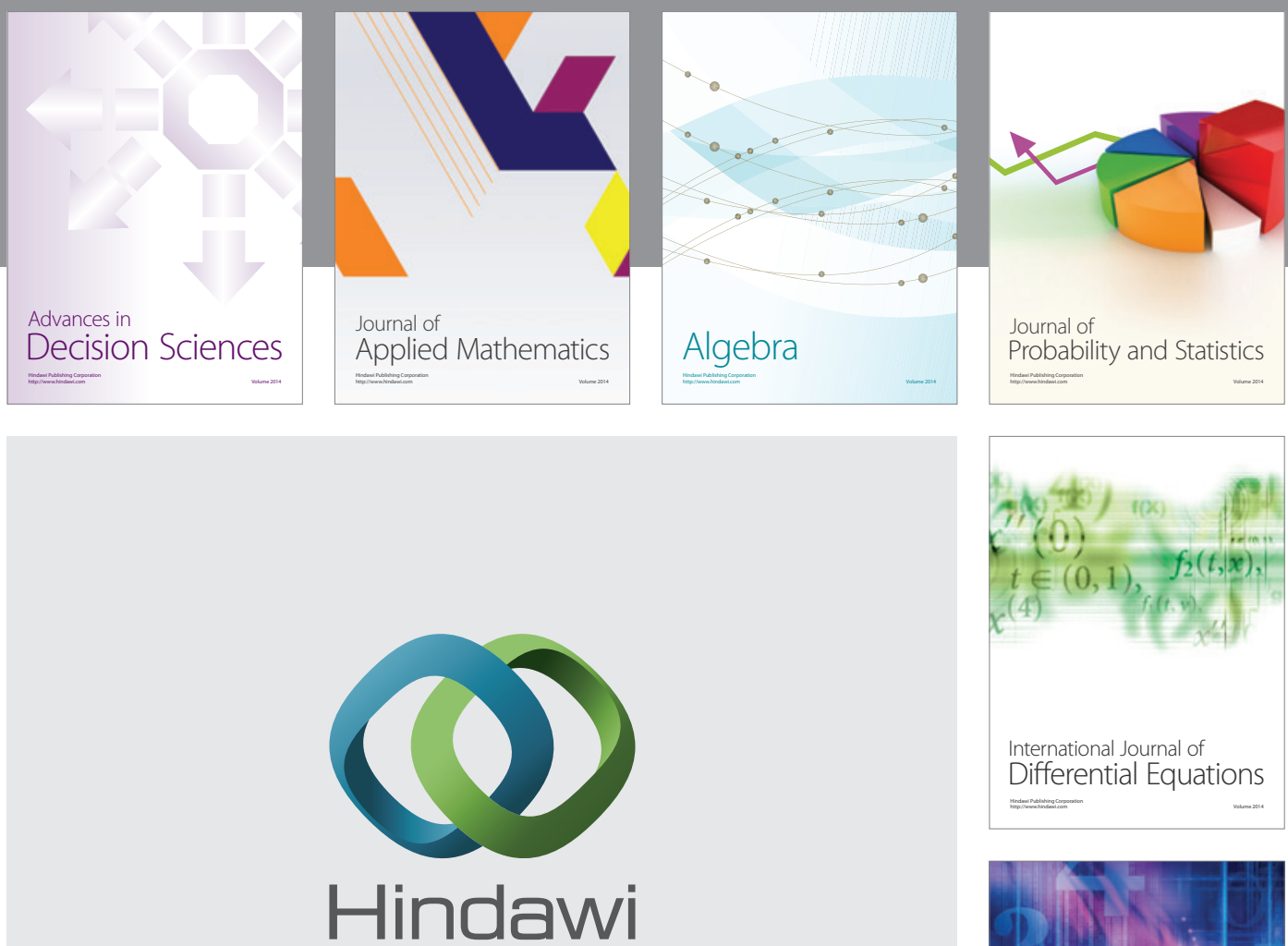

Submit your manuscripts at http://www.hindawi.com
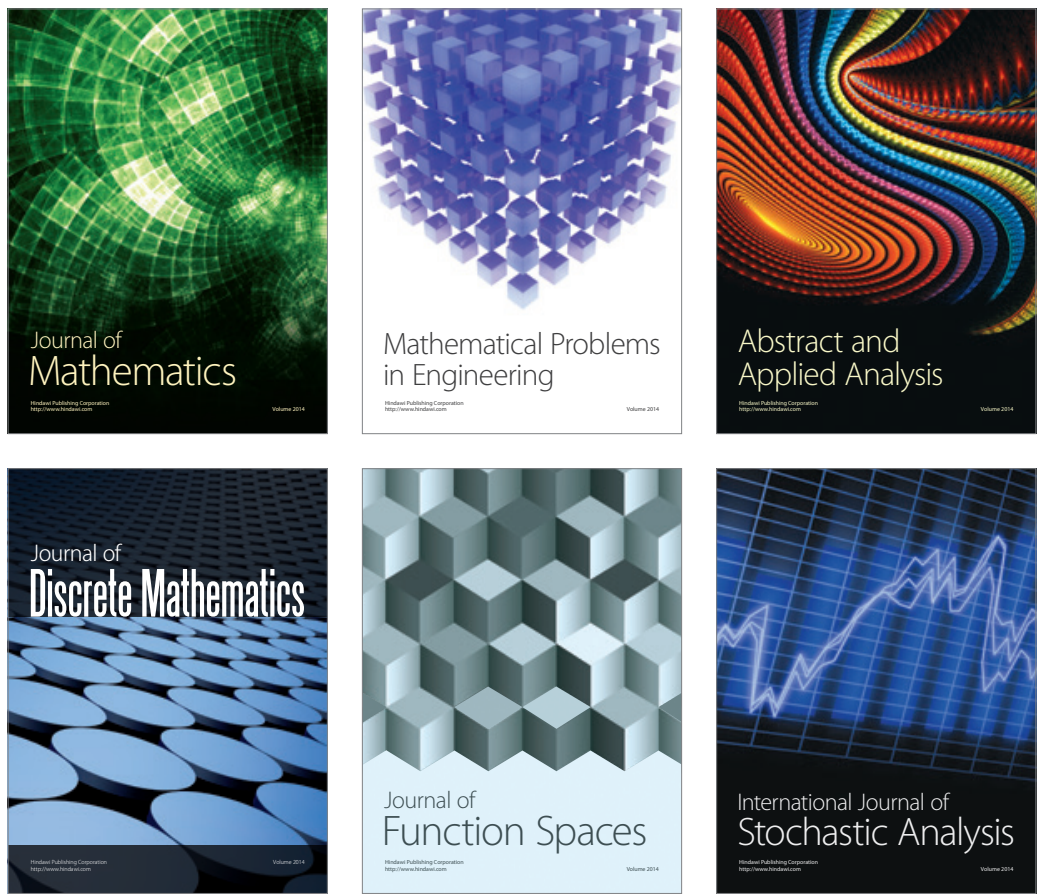

Journal of

Function Spaces

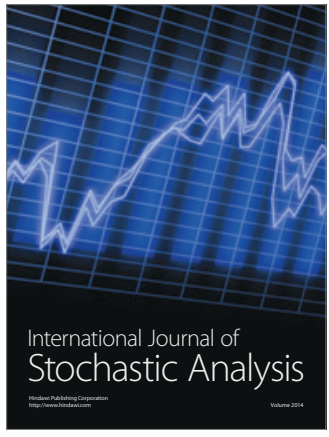

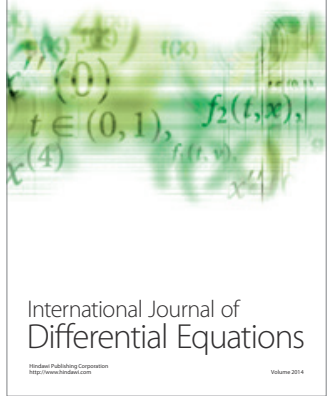
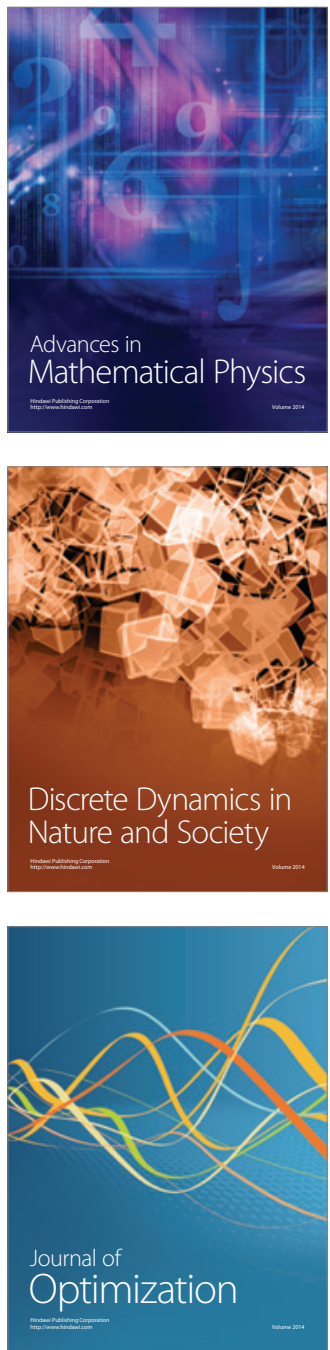